\title{
Geometric Design-based Dimensional Synthesis of a Novel Metamorphic Multi-fingered Hand with Maximal Workspace
}

Wei An ${ }^{1}$, Jun Wei ${ }^{1}$, Xiaoyu Lu ${ }^{1 *}$ C, Jian S. Dai ${ }^{1,2}$ and Yanzeng $\mathrm{Li}^{3}$

\begin{abstract}
Current research on robotic dexterous hands mainly focuses on designing new finger and palm structures, as well as developing smarter control algorithms. Although the dimensional synthesis of dexterous hands with traditional rigid palms has been carried out, research on the dimensional synthesis of dexterous hands with metamorphic palms remains insufficient. This study investigated the dimensional synthesis of a palm of a novel metamorphic multifingered hand, and explored the geometric design for maximizing the precision manipulation workspace. Different indexes were used to value the workspace of the metamorphic hand, and the best proportions between the five links of the palm to obtain the optimal workspace of the metamorphic hand were explored. Based on the fixed total length of the palm member, four nondimensional design parameters that determine the size of the palm were introduced; through the discretization method, the influence of the four design parameters on the workspace of the metamorphic hand with full-actuated fingers and under-actuated fingers was analyzed. Based on the analysis of the metamorphic multi-fingered hand, the symmetrical structure of the palm was designed, resulting in the largest workspace of the multi-fingered hand, and proved that the metamorphic palm has a massive upgrade for the workspace of underactuated fingers. This research contributed to the dimensional synthesis of metamorphic dexterous hands, with practical significance for the design and optimization of novel metamorphic hands.
\end{abstract}

Keywords: Metamorphic hands, Dimensional synthesis, Maximal workspace, Discretization

\section{Introduction}

For decades, robotic and prosthetic hand designers have been interested in making a life-like mechanical dexterous hand, from which a large number of robotic hands can be derived. To prevent the problems caused by the freedom of a human hand as much as possible, researchers and designers use under-actuated fingers and palms with low degrees of freedom to achieve grasping action [1]. Simpler hands with fewer joints and fingers can still achieve a high degree of functionality with a simplified structure [2]. With the development of computer

\footnotetext{
*Correspondence: Ixy_0826@tju.edu.cn

1 MOE Key Laboratory for Mechanism Theory and Equipment Design, International Centre for Advanced Mechanisms and Robotics, Tianjin University, Tianjin, China

Full list of author information is available at the end of the article
}

technology, as well as control and institutional theories, scientists have started studying robotic dexterous hands systematically, leading to the emergence of various dexterous hands that can imitate the structure of human hands. Consider the example of a typical Okada hand [3] developed in Japan; its three fingers are capable of mimicking a human hand.

With further advancement in the field, several famous robot dexterous hands [4-10] have been created. As for various notable designed hands, the three-fingered Stanford/JPL Hand [4], four-fingered Utah/MIT Hand [5], five-fingered Belgrade/USC hand [6], Karlsruhe Dexterous IN Hand [7], DLR hand [8], NAIST Hand [9], and UBH3 hand [10] have provided pioneering insights. In the 21st century, international research on robotic dexterous hands has gradually shifted from exploration and 
research to practical application development. Meanwhile, the application range has been expanding, and an increasing number of dexterous hands have appeared in various industries. $\mathrm{Li}$ et al. [11] designed a novel deployable grasping manipulator (DGM). Zhao et al. [12] proposed an $n$ (3RRIS) metamorphic serial-parallel manipulator (MSPM) with multiple working conditions. Jin et al. [13] developed dexterous hands based on parallel finger structures, and Fang et al. [14] presented a novel Lie-group-based synthesis method that extends the $3-[\mathrm{P}][\mathrm{S}]$ parallel mechanisms (PMs) to dexterous hands. For the uncontrollable problem of underactuated dexterous hand joint movement, a novel lockable joint singleturn underactuated dexterous hand was designed by Liu et al. [15]. Laffranchi et al. [16] created the Hannes hand. Gong et al. [17] developed a dexterous anthropomorphic robotic hand by mimicking the muscle-tendon-ligament structure of the human hand. Wang et al. [18] proposed a grasper composed of rigid mechanical structures, which was able to drive and control with a single degree of freedom and automatically self-adapts to the object shape with a uniform grasping force. Wang et al. [19] revealed a passively adaptive five-fingered underactuated dexterous hand (UADH). Yang et al. [20] presented an anthropomorphic robotic hand (MCR-Hand III). Yang et al. [21] developed a rigid-flexible coupling bionic finger based on the structure of the human hand. Zhang et al. [22] designed a novel linear-parallel and self-adaptive robot hand, and also developed the MPJ hand [23] , the PASA hand [24] and the TPM hand [25]. Loai et al. [26] designed a variable stiffness, soft, three-fingered dexterous gripper. Glick et al. [27] created a soft robotic gripper with a gecko-inspired adhesive.

To overcome the negative effects of low palm flexibility on the workspace, operation, and dexterity of robotic hands, Dai et al. [28, 29] applied the variable topological structure of the metamorphic mechanism to extend the degree of freedom of the palm, which addressed the limitation of the low flexibility of the traditional dexterous palm and creatively proposed the design concept of a dexterous hand. The palm of the metamorphic hand was based on a closed-loop spherical five-bar mechanism that can be folded and unfolded. The fingers were mounted on the components of the spherical mechanism. The position of the finger end can be adjusted via the palm movement, which greatly increased the reach of the finger and expanded the workspace of the entire hand. Similarly, the use of a metamorphic mechanism in the design of the palm significantly improved the dexterity and imitation of the robot hand, and achieved more general functions in its application.

Many studies have been conducted to examine the design parameters of robotic hands and how they affect the grasping performance. Ciocarlie and Allen [30] used a quasistatic equilibrium formulation to predict the stability of a given grasp. Ciocarlie et al. [31] introduced a method to optimize the dimensions of the links to achieve an enveloping grasp of a large range of objects. Borràs and Dollar [32] applied dimensional synthesis to explore the geometric design of dexterous three-fingered robotic hands to maximize the precision manipulation workspace. They also found that proper parameter design for an underactuated hand can achieve up to $50 \%$ of the workspace of a fully actuated hand. Roshan and Kumar [33] presented the design exploration of robotic hands to achieve a maximum precision manipulation workspace.

Although many dimensional syntheses have been applied to mechanical hands to achieve better workspace and grip performance, there exist few studies regarding the application of dimensional synthesis in metamorphic hands. Cui and Dai [34] designed a metamorphic hand with a planar reconfigurable and flexible palm, making the hand more dexterous and adaptable. Nevertheless, they merely analyzed the kinematics of the palm and ignored the kinematics of the fingers. This study aims to investigate the dimensional synthesis of a metamorphic hand and discuss the effects of the design parameters on the palm and finger workspaces. In addition, this work also investigates the necessity of a symmetrical structure in the palm for maximal workspace, and how the metamorphic mechanism in the palm changes the size of the finger workspace in the metamorphic hand. Based on the powerful performance of the original metamorphic hand [34], this study has made great progress, whose conclusion could be suitable for further exploration of other metamorphic hands. The method for optimizing the design parameters of the metamorphic hand is also significant.

The rest of this article is organized as follows: the mathematical formulation of the hand is presented in Section 2. The subsequent Section 3 outlines the methodology for systematically computing the workspaces and their size. The computation results and relevant discussions are elaborated in Section 4, while Section 5 presents the conclusions.

\section{Kinematics and Workspace of the Metamorphic Multi-fingered Hand}

A three-fingered dexterous hand was developed with a planar five-bar link mechanism as its reconfigurable palm, as illustrated in Figure 1. This metamorphic hand consists of two parts: the palm and fingers. The palm is comprised of five links (links 1-5) that are connected in a sequence. There exist three identical double-joint fingers (fingers 1-3, as shown in Figure 1) installed on the palm, which are fixated on links 2,3 , and 5 , respectively. 


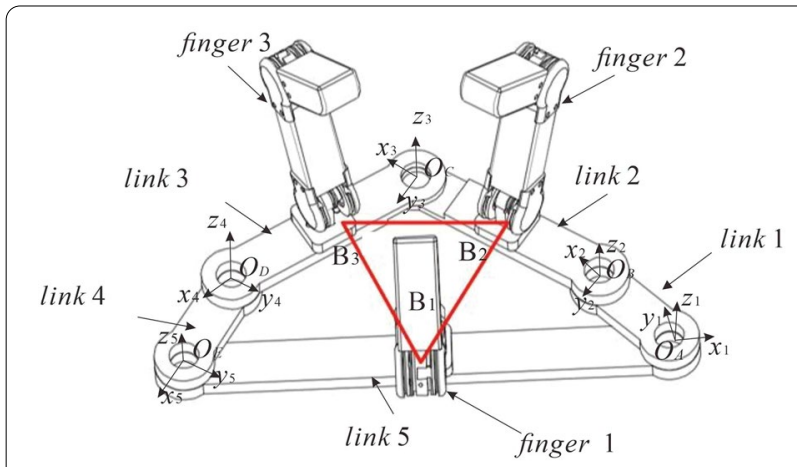

Figure 1 Workspace triangle of the metamorphic hand palm

Considering that the palm configuration could be expressed by a triangle determined by the fixing points of the fingers, namely $B 1, B 2$, and $B 3$ in Figure 1, this study defined the triangle area as the palm workspace as per Cui and Dai [34] to apply dimensional synthesis on the novel metamorphic multi-fingered hand. With the given input angles of the palm portion, the area of the triangle, $\triangle B 1 B 2 B 3$ can be used to evaluate the workspace of the palm [28]. If the triangular area in the working area of the palm is large, it indicates that the palm is widely opened, and can grasp a large object. In the case of a small triangular area in the workspace, it is suitable for holding a small object. In addition, this study defined the space within the reach of the fingertip as the finger workspace.

\subsection{Mathematical Model for Kinematics of the Metamorphic Multi-fingered Hand}

The schematic of the metamorphic hand explains the mathematical relationship of its reconfigurable palm, as shown in Figure 1. The global coordinate frame, $O_{A} x_{1} y_{1} z_{1}$ is fixed with respect to link 5 , where $z_{1}$ is the axis of the revolute joint, $O_{A}$, and $x_{1}$ lies along link 5. Similarly, local coordinate frames, $O_{B} x_{2} y_{2} z_{2}, O_{C} x_{3} y_{3} z_{3}, O_{D} x_{4} y_{4} z_{4}$, and $O_{E} x_{5} y_{5} z_{5}$ are established with respect to links 1 to 4 , respectively, where $z_{i}(i=2-5)$ is the axis of each palm revolute joint, and $x_{i}$ lies along links $1-4 . \theta_{i}(i=1-5)$ is the angle between two connecting links where $\theta_{1}$ and $\theta_{5}$ are actuated joints, while $\theta_{2}, \theta_{3}$, and $\theta_{4}$ are passive joints that change the configuration of the metamorphic palm in the company. Cui and Dai [34] established a kinematic model of the metamorphic hand and obtained some formulas for its forward and inverse kinematics as follows:

$$
\theta_{2}=2 \arctan \left(\frac{B_{1} \pm \sqrt{A_{1}^{2}+B_{1}^{2}-C_{1}^{2}}}{A_{1}-C_{1}}\right)-\theta_{1}
$$

$$
\begin{aligned}
& \theta_{4}=2 \arctan \left(\frac{B_{2} \pm \sqrt{A_{2}^{2}+B_{2}^{2}-C_{2}^{2}}}{A_{2}-C_{2}}\right)-\theta_{5}, \\
& \theta_{3}=2 \pi-\theta_{1}-\theta_{2}-\theta_{4}-\theta_{5},
\end{aligned}
$$

where

$$
\begin{aligned}
& A_{1}=2\left(\mathrm{c} \theta_{1} l_{1}+\mathrm{c} \theta_{5} l_{4}+l_{5}\right) l_{2}, \\
& B_{1}=2\left(\mathrm{~s} \theta_{1} l_{1}-\mathrm{s} \theta_{5} l_{4}\right) l_{2}, \\
& C_{1}=\left(\mathrm{c} \theta_{1} l_{1}+\mathrm{c} \theta_{5} l_{4}+l_{5}\right)^{2}+\left(\mathrm{s} \theta_{1} l_{1}-\mathrm{s} \theta_{5} l_{4}\right)^{2}+l_{2}^{2}-l_{3}^{2}, \\
& A_{2}=2\left(\mathrm{c} \theta_{1} l_{1}+\mathrm{c} \theta_{5} l_{4}+l_{5}\right) l_{3}, \\
& B_{2}=2\left(\mathrm{~s} \theta_{5} l_{4}-\mathrm{s} \theta_{1} l_{1}\right) l_{3}, \\
& C_{2}=\left(\mathrm{c} \theta_{1} l_{1}+\mathrm{c} \theta_{5} l_{4}+l_{5}\right)^{2}+\left(\mathrm{s} \theta_{1} l_{1}-\mathrm{s} \theta_{5} l_{4}\right)^{2}+l_{3}^{2}-l_{2}^{2} .
\end{aligned}
$$

For the configuration of the actual dexterous hand, the palm is generally in the configuration of a convex pentagon when grasping, so the solutions of $\theta_{2}$ and $\theta_{4}$ in the above formulas are positive.

\subsection{Workspace of the Metamorphic Palm}

Cui and Dai [34] conducted a preliminary study of the workspace of metamorphic palms. The input angle, $\theta_{1}$ was fixed, with another input angle, $\theta_{5}$ changed from $\pi / 2$ to $3 \pi / 2$ to study the orientation of the fingers.

The workspace of the palm has a vast range. It can be seen that the metamorphic palm has a variable workspace so that the size of the fingers and palm should be explored to obtain an optimal metamorphic hand. In this study, all the input joints were actuated, while many poses of the metamorphic hand were obtained to explore the maximum workspace of the palm, and dimensional synthesis was applied to the metamorphic palm.

\subsection{Workspace of Fingers of the Metamorphic Multi-fingered Hand}

The workspace of the fingers is combined with that of the palm, as the fingers are fixed on the links. In this study, the metamorphic hand is also a three-fingered robot hand, and thus, the conclusions of Borràs and Dollar [32] can be applied. To maximize the workspace of the metamorphic hand, the distal link of the two-link fingers should reach approximately 1-1.2 times of the proximal link in terms of the length.

The novel metamorphic hand is characterized by a variable workspace of the palm that can extend the 




Figure 2 Novel metamorphic multi-fingered hand grasping a disk

posture of the three identical fingers, thereby increasing the dexterity of grasping. To explore the workspace of the fingers, a grasp model of the metamorphic hand was established based on the precision grasp. Therefore, a scheme for the metamorphic hand grasping a disc could be developed, as shown in Figure 2.

To establish a gripping triangle model of the novel dexterous hand, the first task was to establish a position model of the fingertips. Finger 1, which is fixed on the base, is not affected by changes in the palm.

$$
\left\{\begin{array}{l}
x_{C_{1}}=-l_{5} / 2, \\
y_{C_{1}}=-f_{1} \cos \left(\varphi_{11}\right)-f_{2} \cos \left(\varphi_{11}+\varphi_{12}\right), \\
z_{C_{1}}=f_{1} \sin \left(\varphi_{11}\right)+f_{2} \sin \left(\varphi_{11}+\varphi_{12}\right),
\end{array}\right.
$$

where $x_{C i}, y_{C i}, z_{C i}(i=1,2,3)$ are the coordinates of the three fingertips, respectively, and $\phi_{i j}(i=1,2,3 ; j=1,2)$ represents the angle of the joint, $j$ in the fingers, $i . f_{1}$ and $f_{2}$ represent the lengths of the proximal and distal links, respectively.

For finger 2, which is fixed on link 2, the relationship can be obtained as follows:

$$
\begin{aligned}
& p_{\mathrm{C}_{2}}=R\left(z_{1}, \theta_{1}\right) T\left(x_{1}, l_{1}\right) R\left(z_{2}, \theta_{2}\right) T\left(x_{2}, l_{2} / 2\right) \\
& R\left(x_{2},-\varphi_{21}\right) T\left(y_{2},-f_{1}\right) R\left(x_{2},-\varphi_{22}\right) T\left(y_{2},-f_{2}\right){ }_{\mathrm{C}_{2}} p_{\mathrm{C}_{2}}, \\
& O_{\mathrm{C}_{2}} p_{\mathrm{C}_{2}}=\left[\begin{array}{llll}
0 & 0 & 0 & 1
\end{array}\right]^{\mathrm{T}} .
\end{aligned}
$$

Therefore, the coordinates of the second fingertip are as follows:

$$
\begin{gathered}
x_{\mathrm{C}_{2}}=l_{1} \mathrm{c} \theta_{1}+l_{2} \mathrm{c}\left(\theta_{1}+\theta_{2}\right) / 2+ \\
\mathrm{s}\left(\theta_{1}+\theta_{2}\right)\left(f_{1} \mathrm{c} \varphi_{21}+f_{2} \mathrm{c}\left(\varphi_{21}+\varphi_{22}\right)\right), \\
y_{\mathrm{C}_{2}}=l_{1} \mathrm{~s} \theta_{1}+l_{2} \mathrm{~s}\left(\theta_{1}+\theta_{2}\right) / 2- \\
\mathrm{c}\left(\theta_{1}+\theta_{2}\right)\left(f_{1} \mathrm{c} \varphi_{21}+f_{2} \mathrm{c}\left(\varphi_{21}+\varphi_{22}\right)\right), \\
z_{\mathrm{C}_{2}}=f_{1} \sin \varphi_{21}+f_{2} \sin \left(\varphi_{21}+\varphi_{22}\right) .
\end{gathered}
$$

The coordinates of the third fingertip can also be obtained similarly:

$$
\begin{gathered}
x_{\mathrm{C}_{3}}=-l_{5}-l_{4} \mathrm{c} \theta_{5}-l_{3} \mathrm{c}\left(\theta_{4}+\theta_{5}\right) / 2 \\
-\mathrm{s}\left(\theta_{4}+\theta_{5}\right)\left(f_{1} \mathrm{c} \varphi_{31}+f_{2} \mathrm{c}\left(\varphi_{31}+\varphi_{32}\right)\right), \\
y_{C_{3}}=l_{4} \mathrm{~s} \theta_{5}+l_{3} \mathrm{~s}\left(\theta_{4}+\theta_{5}\right) / 2 \\
-\mathrm{c}\left(\theta_{4}+\theta_{5}\right)\left(f_{1} \mathrm{c} \varphi_{31}+f_{2} \mathrm{c}\left(\varphi_{31}+\varphi_{32}\right)\right), \\
z_{\mathrm{C}_{3}}=f_{1} \sin \varphi_{31}+f_{2} \sin \left(\varphi_{31}+\varphi_{32}\right) .
\end{gathered}
$$

These three points were connected to form a grasping triangle. On this basis, the range of the grasp space and size range of the grasped object can be further analyzed.

As shown in Figure 3 , points $C_{1}, C_{2}$, and $C_{3}$ are the gripping points, with point $P_{\mathrm{O}}$ indicating the object center. For the disc model constrained by the three fingertips, the center position of the grasped object is the best indicator of the position of the grasped object, while the distance from the three gripping points to the center of the circle is the best indicator of the size of the grasped object. Meanwhile, the vector direction of the three gripping points and the center position coordinates are the best indicators of the degree of inclination of the grasped object.

\section{Dimensionless Optimizations and Discretization of the Metamorphic Multi-fingered Hand \\ 3.1 Parametric Design of the Metamorphic Multi-fingered Hand}

The design significance of the dexterous hand lies in its ability to complete the target grasping task of an actual object, while the quality of task completion depends on the flexibility and operability of the grasp. For a dexterous hand, changes in the palm-size and finger-size parameters affect the topological characteristics of the entire dexterous hand, which in turn affects the kinematics, gripping characteristics, and target tasks of the entire dexterous hand, and work performance.

Therefore, for different grabbing tasks, there is always a new dexterous hand of a specific size that allows it to have maximum grip flexibility or grip operability under the constraints of the gripping scheme and target. Therefore, the targeted size optimization of the

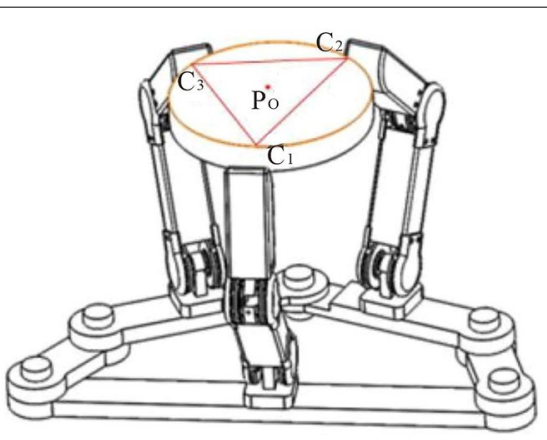

Figure 3 Center of the grasped object 
dexterous hand can lay a hardware foundation to better meet the requirements of actual grab tasks, reduce the subsequent sensor and control accuracy requirements, and increase the grabbing range of the object under specific working conditions, as well as improving the grab stability and performance of target grabbing tasks.

Based on the different gripping attributes required for the target grasping task, the goals of dexterous hand size optimization can be classified into two categories. One is to obtain the best grip flexibility for the target being grasped, and the other is to obtain the best grip operability for the target being grasped.

The first target type is focused more on the breadth of the grasping scope, the richness of the grasping posture, and the adaptability of the size of the grasped object. From the perspective of static characteristics, such as position and attitude, it seeks the extreme value of the gripping flexibility of the new metamorphic hand under the grasping task.

For the second type of target, it is aimed at the target grabbing task, the stability of the palm-handling operation, and the mechanical and control performances of the continuous gripping and operating motions. It mainly seeks the extreme value of the grasping operability of the new type of changes under the grasping task from the perspective of dynamic characteristics, such as the Jacobian matrix.

To optimize the size of the new dexterous hand, the grip performance basis of the size optimization is selected in terms of the focus function of the grab task, while establishing the optimization model. The optimization model consists of two main parts: the objective function and constraints.

To standardize the results of all different sizes of metamorphic hands, the units of the parameters are omitted herein. Considering that the final size is as close as possible to the previous generation of metamorphic hands, the sum of the lengths of the palm members should be $\sim 35 \mathrm{~cm}$. Therefore, the sum of the lengths of the five links of the palm is fixed to $35 \mathrm{~cm}$. Link 5 is used as the base length with respect to the longest among the five links. Through the length proportion between the different links, the length of the five links can be determined, and the ratio between these links can be represented as four parameters. In this study, the lengths of the links are in centimeters.

The parameters described in Table 1 are as follows: $P_{1}=l_{5} / l_{1}$ : The ratio of the length of link $l_{5}$ to that of link $l_{1}$ of the palm; specifically, $P_{i}$ represents the ratio of link $l_{5}$ to link $l_{\mathrm{i}}$. Because of the structural limits of the reconfigurable palm, the value of $P_{i}$ should be limited, and the ranges of the four parameters are listed in Table 1.
Table 1 Design parameters and their corresponding ranges

\begin{tabular}{lll}
\hline Parameter & Definition & Range \\
\hline$P_{1}$ & $I_{5} / I_{1}$ & $2-8$ \\
$P_{2}$ & $I_{5} / I_{2}$ & $0-2.5$ \\
$P_{3}$ & $I_{5} / I_{3}$ & $0-2.5$ \\
$P_{4}$ & $I_{5} / I_{4}$ & $2-8$ \\
\hline
\end{tabular}

If $P_{i}$ is too large, the configuration of the palm would be similar to a line, and the metamorphic characteristics of the palm would be weakened. If $P_{1}$ and $P_{4}$ are $<2$, with $\theta_{1}$ and $\theta_{5}$ taking the values of $\pi$ and 0 , respectively, the area of the palm triangle would become zero, or even a negative value. Thus, the ranges of $P_{1}$ and $P_{4}$ should be $>2$.

$P_{i}$ defines the geometry of the palm, which is sufficient for calculating the kinematic workspace that is defined by solving the kinematics. They are sufficient for computing the workspace of metamorphic palms. To determine the size of the palm workspace with any combination of four different design parameters and represent the results graphically, these parameters need to be discretized. A small step size is taken for each design parameter within a reasonable range, and the palm workspace is solved numerically for all possible combinations.

\subsection{Discretization Method of Design Parameters of the Metamorphic Multi-fingered Hand}

While solving the combination of any four design parameters, a discretization method should be employed. The two active angles, $\theta_{1}$ and $\theta_{5}$, of the palm take a small step size within the range of motion to solve the workspace of the dexterous palm, where $\theta_{1}$ and $\theta_{5}$ have a value range of $[0, \pi]$.

In the case where the discretization method is used to calculate the workspace of the entire palm under the design parameters, smaller step size of the variable leads to a more accurate result. However, a small step size corresponds to a large increase in calculations, which is nonlinear because the palm has two active joints. A proper step size should be found to ensure that the calculated results are as close as possible to the actual situation, while not significantly increasing the computing time.

Based on the original version of the dexterous palm size, this study solves the error of the palm workspace with different input angle steps, as shown in Table 2.

Where $S_{\max }$ and $S_{\text {ave }}$ indicate the maximum and average areas of the palm triangle in the entire motion space, respectively. In this study, $0.001 \pi$ is taken as the ideal step size, and a comparison of the results of other groups with different step sizes is made with the seventh group to determine the error. In the fourth set of data, if the step size is $0.01 \pi$, more than 10000 poses in the dexterous 
Table 2 Error in different design parameters

\begin{tabular}{llccccc}
\hline & $\boldsymbol{\theta}_{\mathbf{1}}$ and $\boldsymbol{\theta}_{\mathbf{5}}$ step size & $\begin{array}{l}\text { Number of } \\
\text { configurations }\end{array}$ & $\boldsymbol{S}_{\max }\left(\mathbf{c m}^{2}\right)$ & $\boldsymbol{S}_{\text {ave }}\left(\mathbf{c m}^{2}\right)$ & $\boldsymbol{S}_{\max }$ error (\%) & $\boldsymbol{S}_{\text {ave }}$ error (\%) \\
\hline 1 & $0.1 \pi$ & 121 & 12.424 & 9.617 & 1.18571 & 3.75545 \\
2 & $0.05 \pi$ & 441 & 12.564 & 9.714 & 0.07171 & 2.78372 \\
3 & $0.025 \pi$ & 1681 & 12.564 & 9.871 & 0.07171 & 1.20782 \\
4 & $0.01 \pi$ & 10201 & 12.573 & 9.951 & 0.00138 & 0.41380 \\
5 & $0.005 \pi$ & 40401 & 12.573 & 9.975 & 0.00138 & 0.16398 \\
6 & $0.0025 \pi$ & 160081 & 12.573 & 9.985 & $0.00066 \%$ & 0.06559 \\
7 & $0.001 \pi$ & 1002001 & 12.573 & 9.992 & - & - \\
\hline
\end{tabular}

palm movement space can be obtained, and the calculated result has an error of less than 1\% compared with the ideal result. Therefore, in the subsequent dimension synthesis calculation, a step size of $0.01 \pi$ is suitable for simulating the entire motion space for a set of design parameters.

\section{Results of Dimensional Synthesis of the Metamorphic Multi-fingered Hand}

4.1 Dimensional Synthesis of Palm Workspace Indicators with Different Parameters of the Metamorphic Multi-fingered Hand

In this study, dimensional synthesis is applied to the metamorphic multi-fingered palm with different palm workspace indicators, and observations are made on the relationship between the design parameters and the indicators. The indicators include:

a. The maximum area of the palm triangle that can be reached, namely, $S_{\max }$.

b. The average area of the palm triangle in the entire motion space, that is, $S_{\text {ave }}$.

c. The arithmetic mean of $S_{\max }$ and $S_{a v e}$, namely, $S^{\prime}$.

The palm of the original metamorphic multi-fingered hand is of a symmetrical structure if the actuated joints $\theta_{1}$ and $\theta_{5}$ are equal. In the original version of the dexterous hand, the length of link 1 is equal to that of link 4 , and the length of link 2 is equal to that of link 3 , which means that the parameters in Table 1 can be reduced to two parameters $P_{1}$ and $P_{2}$. However, the asymmetry of the palm can affect the workspace of the palm. To explore the effect of the asymmetry of the distribution of link length in the workspace of the metamorphic palm, the total length was fixed, while $P_{1}$ and $P_{4}$, as well as $P_{2}$ and $P_{3}$ were varied, and were changed from the lower to the upper limit value. An independent simulation was performed by sweeping all the parameters in the ranges shown in Table 3.
Table 3 Palm design parameters

\begin{tabular}{lllc}
\hline Design parameters & Range & Step size & $\begin{array}{l}\text { Number } \\
\text { of values }\end{array}$ \\
\hline$P_{1}$ & {$[2,8]$} & 0.05 & 121 \\
$P_{2}$ & 0.5 & 5 \\
$P_{3}$ & {$[0,2.5]$} & 0.5 & 5 \\
$P_{4}$ & {$[0,2.5]$} & 0.05 & 121 \\
\hline
\end{tabular}

Because the final results are affected by four variables, it is difficult to reflect the exact results directly in a graphical manner. In this study, a larger step size was used for the overall large range, the best area was determined, and then a detailed analysis of the area was performed to obtain the best results.

The workspace of the palm with different $P_{i}$ values was determined and presented in one picture. If the length of link 1 and that of link 4, as well as the length of link 2 and that of link 3 take the same value, the workspace of the palm can achieve the maximum value with the total length of the palm fixed. More importantly, the average workspace of the metamorphic palm could also reach its maximum. Therefore, dimensional synthesis on the metamorphic multi-fingered hand can be applied on the premise that the length of link 1 is equal to that of link 4, and the length of link 2 is equal to that of link 3 . The map is illustrated in Figure 4.

Figure 4 illustrates the evolution of the workspace size as the geometric parameters change with different proportions between different links. The color bar on the right represents the evolution of the palm workspace size, in which the brightest color corresponds to the most prominent size, while the darkest color indicates a size of zero.

The point of each subgraph closest to the red color is always at the bottom left corner of the graph, indicating that the maximum value $S_{\max }$ always emerges at the lower-left corner of each subgraph, where the design parameters $P_{1}$ and $P_{4}$ are both 2. A blank portion 


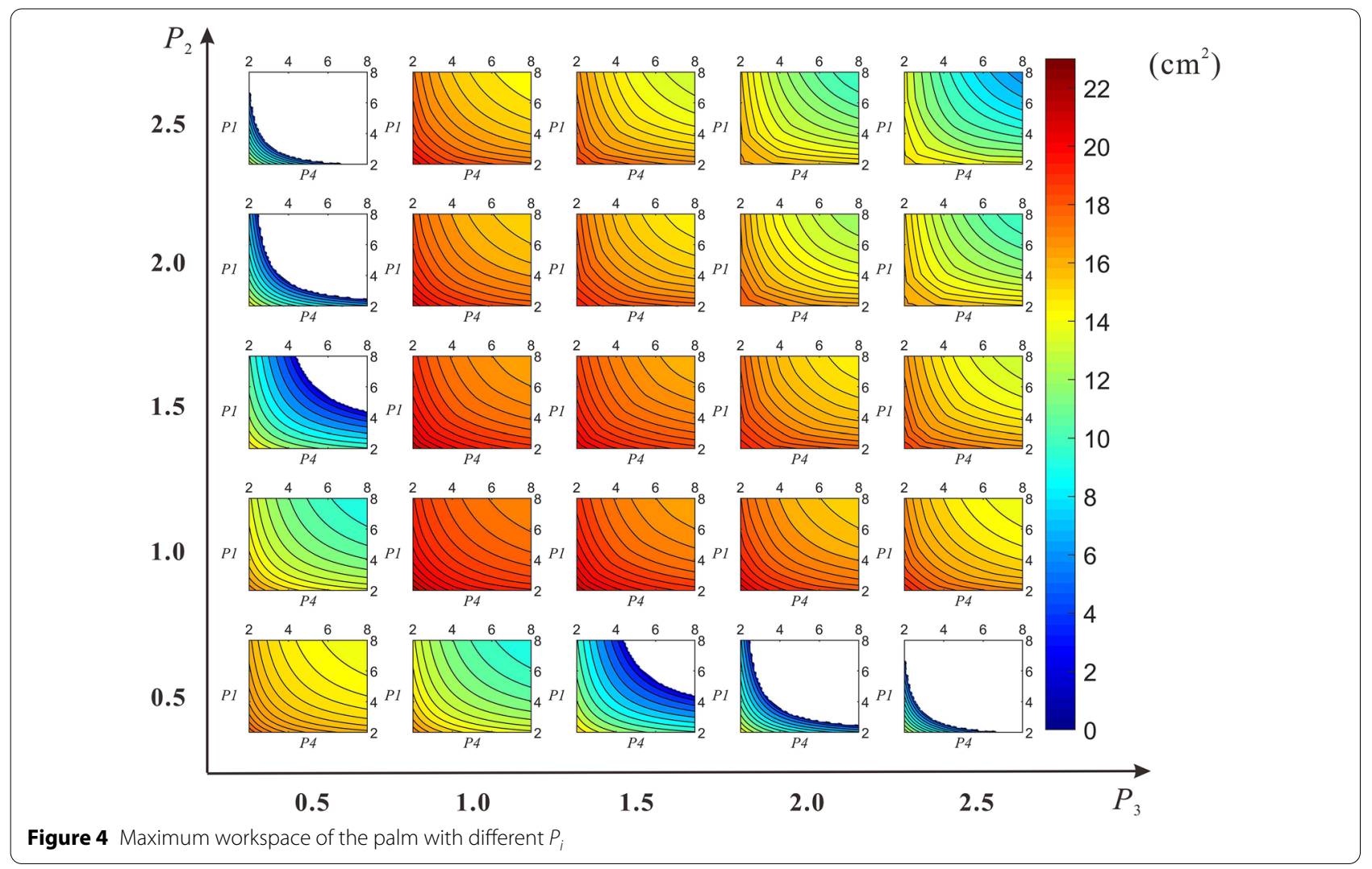

appears in the figure, which indicates that the design parameters corresponding to the blank portion will not constitute the palm of the dexterous hand.

For the average value of the triangular area of the palm over the entire range of motion, the results are as illustrated in Figure 5.

Compared with the results of $S_{\max }$, the change in $S_{\text {ave }}$ only exhibits the same rule as $S_{\max }$ in some graphs. In other subgraphs, the value of $S_{\text {ave }}$ does not increase with the decrease in $P_{1}$ and $P_{4}$; however, we observe that the values of $S_{\text {ave }}$ in these subgraphs are not large enough. The best result is obtained when both $P_{1}$ and $P_{4}$ are 2, which is consistent with the previous results.

For the arithmetic mean $S^{\prime}$ of $S_{\max }$ and $S_{\text {ave }}$ over the entire range of motion, the results are as shown in Figure 6.

The law exhibited by each subgraph is the same as that presented by $S_{\max }$. The maximum result in each subgraph is almost achieved in the lower-left corner of the subgraph, where the design parameters $P_{1}$ and $P_{4}$ are both 2 .

Figures 4, 5, 6 obviously demonstrate that if $P_{1}$ and $P_{4}$, as well as $P_{2}$ and $P_{3}$ take the same value, the maximum and average workspaces of the palm would reach their maximum values.
Based on the above results, for the purpose of this study, the lengths of link 1 and link 4 , as well as the lengths of link 2 and link 3 , take the same value. The workspace of the palm with a smaller step size is used to determine the optimal proportions between link 1 and link 5 , link 2 and link 5 . When the design parameters are reduced to two, smaller steps are used to achieve more accurate results, as shown in Table 4.

After calculating $S_{\text {max }}, S_{a v e}$, and $S$, the following results are obtained with different combinations of $P_{1}$ and $P_{2}$, as illustrated in Figures 7, 8, 9.

It is clear that if both $P_{1}$ and $P_{2}$ take a large value, there is no corresponding value on the stereogram, which means that the metamorphic palm cannot be formed in that case. The largest values of $S_{\text {max }}$ and $S^{\prime}$ are taken when $P_{1}=2$ and $P_{2}=1$. In this case, $S_{\max }$ is approximately $22 \mathrm{~cm}^{2}$, while $S^{\prime}$ is about $17 \mathrm{~cm}^{2}$. However, the largest value $S_{\text {ave }}$ is achieved in the case of $P_{1}=8$ and $P_{2}=1$, where $S_{\text {ave }}$ is approximately $15 \mathrm{~cm}^{2}$. It can be observed from the projection in the upper right that the design parameter $P_{2}$ has a more significant influence on $S_{\text {max }}, S_{a v e}$, and $S^{\prime}$. Regardless of the value of $P_{1}$, the values of $S_{\text {max }}, S_{a v e}$, and $S^{\prime}$ increase rapidly as $P_{2}$ increases, reach their peaks when $P_{2}=1$, and then decrease with the increase in $P_{2}$; the decreasing speed is lower than 


Table 4 Design parameter values of a symmetrical palm

\begin{tabular}{llll}
\hline Design parameters & Range & Step size & $\begin{array}{l}\text { Number } \\
\text { of values }\end{array}$ \\
\hline$P_{1}$ & {$[2,8]$} & 0.01 & 601 \\
$P_{2}$ & {$[0,5]$} & 0.01 & 501 \\
\hline
\end{tabular}

the increasing speed. Meanwhile, it can be seen that if the value of $P_{2}$ is between 0.5 and 1.5, regardless of the value of $P_{1}$, the values of $S_{\text {max }}, S_{\text {ave }}$, and $S^{\prime}$ are all relatively large, almost exceeding $12 \mathrm{~cm}^{2}$, which is consistent with the previous asymmetry structure. From the projection in the lower right, it can be observed that the design parameter $P_{1}$ has less influence on $S_{\text {max }}, S_{a v e}$, and $S^{\prime}$. As $P_{1}$ increases, $S_{\max }$ and $S^{\prime}$ gradually decreases from the maximum value, while $S_{\text {ave }}$ gradually increases from the minimum value. It can be concluded that if the fingerattached links are of the same length, the workspace of the metamorphic palm will achieve the maximum value. This conclusion can also be applied and further explored in other metamorphic hands.

\subsection{Dimensional Synthesis of Workspace for Both Full-Actuated and Under-Actuated Fingers of Metamorphic Palm}

For a traditional dexterous hand, the fingertip position of a two-joint under-actuated finger is a curve in the space; for a full-actuated two-joint finger, its workspace is a fixed plane in the space. To extend the workspace of traditional dexterous hands, researchers have chosen to add joints on the fingers to achieve a larger workspace. Consider the example of the dexterous hand developed by Borràs and Dollar [32]. This undoubtedly increases the difficulty of controlling the dexterous hand. To explore the effect of the metamorphic palm on the workspace of the fingers, the workspace of one finger fixed on link 3 is concluded, while the workspaces of the full-actuated and under-actuated fingers are determined in this study. A scatter plot of the position of the fully actuated fingertips in the space is presented in Figure 10, whose volume is determined using the alphaShape function. Then, volumes of these two fingers are compared, and the rate between these volumes is concluded. An exciting conclusion can be drawn that, unlike traditional rigid palms, the rate between full-actuated and under-actuated fingers can reach a considerable percentage.

In Figure 10, the blue area indicates the workspace of finger 1 , and the remaining two areas indicate the workspaces of fingers 2 and 3, respectively. Because finger 1 is fixed to the base and cannot generate movement relative to the earth, its workspace is a flat surface. The other two fingers are fixed on the metamorphic hand, and their relative position can be changed according to the change in the posture of the hand; thus, the workspace is a threedimensional figure.

Because the workspace of finger 1 is a plane, while those of fingers 2 and 3 are symmetrically equal, the subsequent calculation for the finger workspace volume refers to the workspace of the end position of finger 2, where the range of the active joints $\theta_{1}$ and $\theta_{5}$ is $[0, \pi]$,
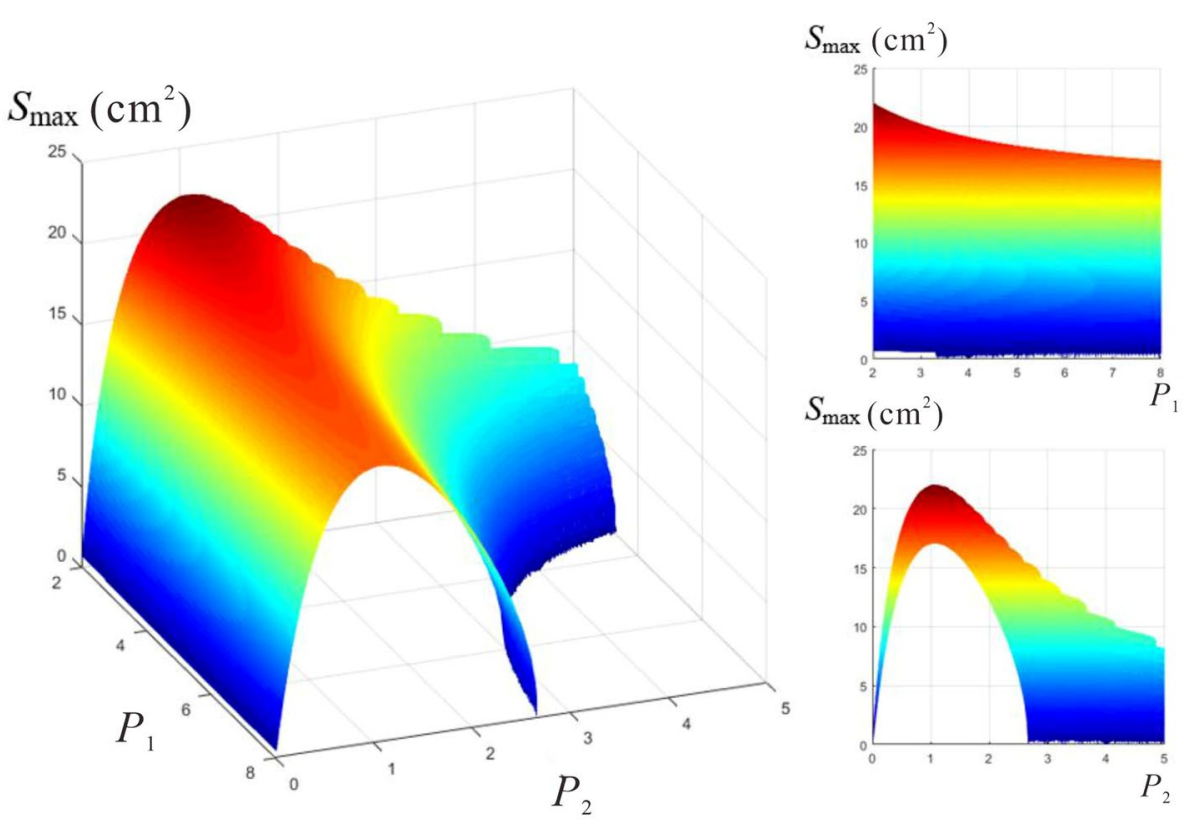

Figure 7 Results of the $S_{\max }$ of the symmetrical palm workspace triangle 



Figure 8 Results of the $S_{\text {ave }}$ of the symmetrical palm workspace triangle

and the range of the two active joints $\theta_{21}$ and $\theta_{22}$ of the finger is $[0, \pi / 2]$.

Through experiments, the appropriate active angle steps can be obtained by considering the final workspace accuracy and computer operation efficiency. The specific parameters are presented in Table 5.

Based on the step sizes in Table 5, the scatter plot of the fingertip position of finger 2 is shown in Figure 11.

In this study, the volume of the finger workspace is taken as a specific indicator to explore the influence of the design parameters on the indicators. Here, three indicators are presented, namely:

a. The volume of the full-actuated finger workspace, $V_{f}$.

b. The volume of the under-actuated finger workspace, $V_{u}$.

c. $V_{r}$, the ratio of $V_{f}$ to $V_{u}\left(V_{r}=V_{u} / V_{f}\right)$.

By calculating 301,101 combinations of different design parameters, $P_{1}$ and $P_{2}$, the following results are obtained, as shown in Figures 12, 13, 14,15.
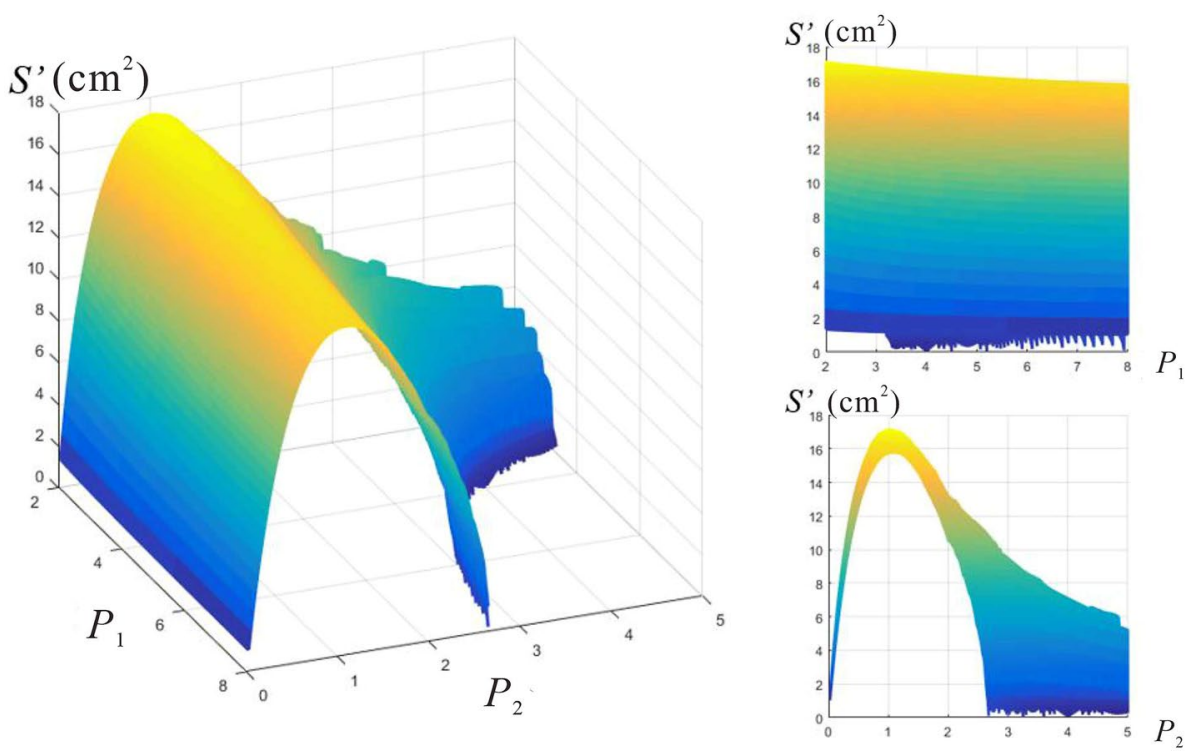

Figure 9 Results of the $S^{\prime}$ of the symmetrical palm workspace triangle 


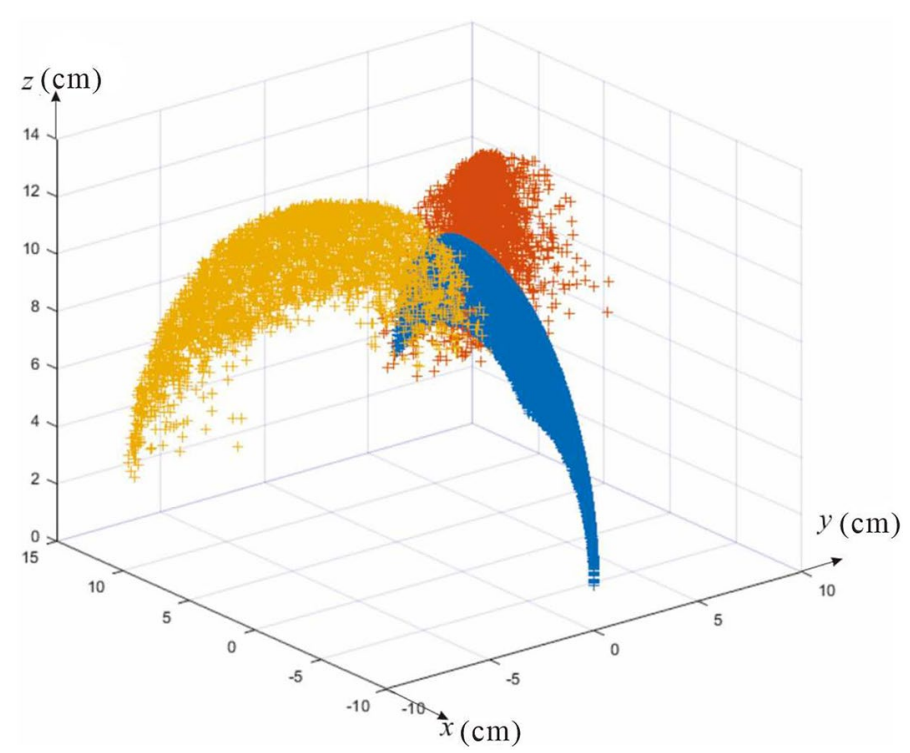

Figure 10 Finger workspace scatter plot of the metamorphic hand

Table 5 Step sizes of the active joints

\begin{tabular}{lllc}
\hline Joints & Step size & Range & Number of values \\
\hline$\theta_{1}$ & $0.02 \pi$ & {$[0, \pi]$} & 51 \\
$\theta_{5}$ & $0.02 \pi$ & {$[0, \pi]$} & 51 \\
$\theta_{21}$ & $0.01 \pi$ & {$[0,0.5 \pi]$} & 51 \\
$\theta_{22}$ & $0.01 \pi$ & {$[0,0.5 \pi]$} & 51 \\
Pose & - & - & 6765201 \\
\hline
\end{tabular}

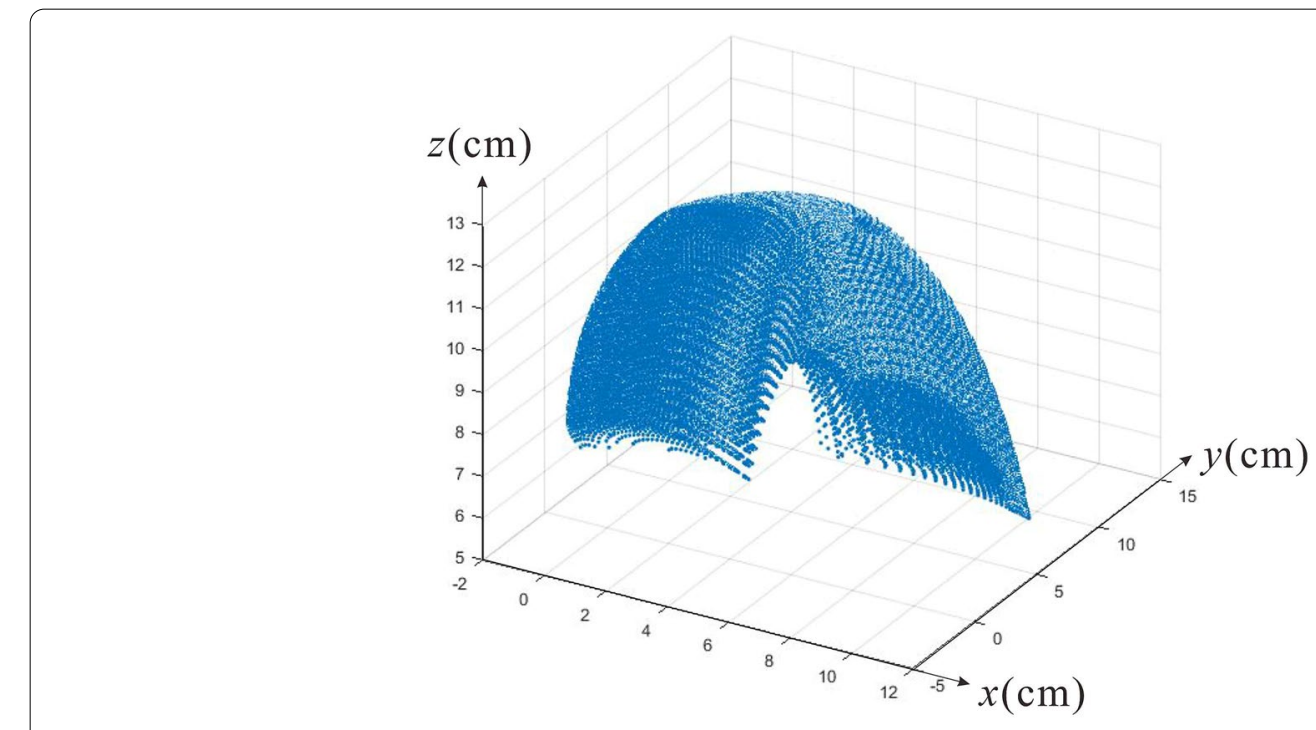

Figure 11 Workspace of the fingertip of finger 2
The under-actuated finger allows one motor to drive multiple joints, reducing the complexity of the control system and lowering costs. For the purpose of this study, the coupling relationship between the two active joints in the finger, $\theta_{i 1}=\theta_{i 2}$ was selected, with $V_{u}$ obtained similarly. The results are as presented in Figure 13.

Figures 12 and 13 demonstrate the relationship between $V_{f}\left(V_{u}\right)$ and the design parameters of the palm. The effect of the palm design parameter, $P_{1}$ on $V_{f}$ and $V_{u}$ is significant without considering $P_{2}$. As $P_{1}$ increases 

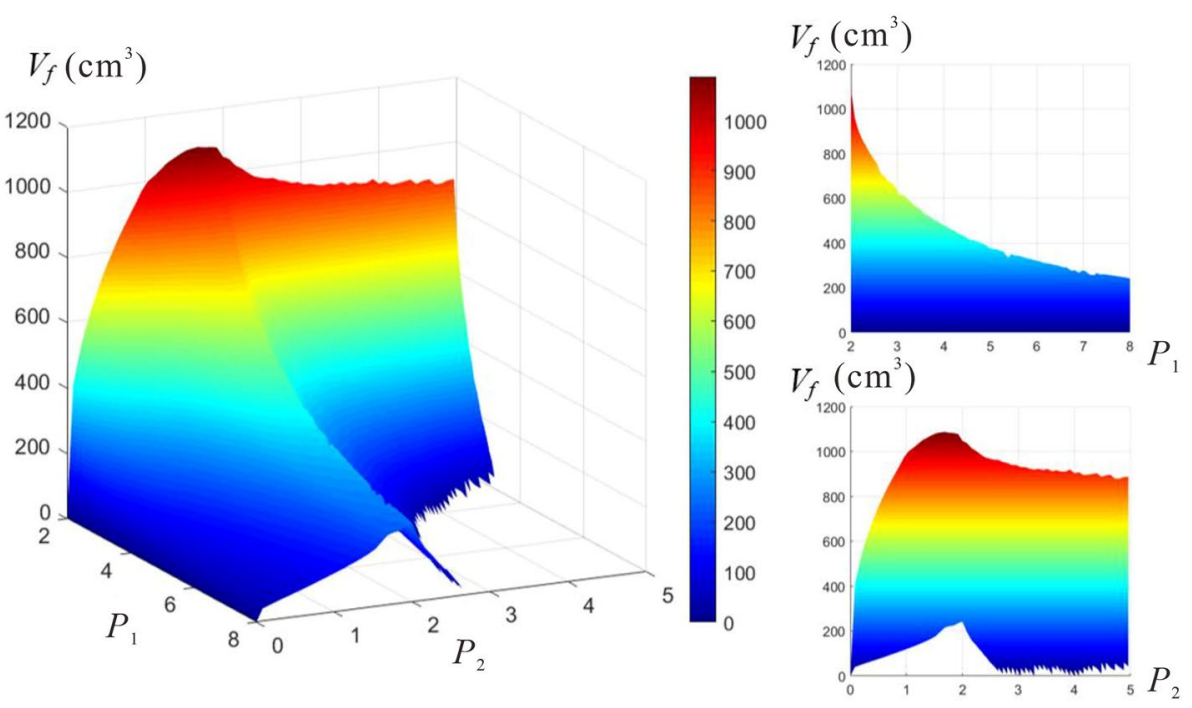

Figure 12 Volume of the workspace of the full-actuated finger 2

from 2 to 8, the sizes of $V_{f}$ and $V_{u}$ gradually decrease with a speed varying from high to low.

Regardless of the palm design parameter $P_{1}$, the sizes of $V_{f}$ and $V_{u}$ tend to increase first and then decrease as $P_{2}$ increases. If the $P_{1}$ is small, the magnitudes of $V_{f}$ and $V_{u}$ continue to increase with $P_{2}$ until the value of $P_{2}$ reaches $\sim 1.7$. Subsequently, the magnitudes of $V_{f}$ and $V_{u}$ gradually decrease as $P_{2}$ increases. Regardless of the value of $P_{1}$ in the range of values, the maximum $V_{f}$ and $V_{u}$ sizes always appear as long as $P_{2}$ remains in the range of $[1,2]$.
The maximum value of $V_{f}$ is achieved when $P_{1}=2$ and $P_{2}=1.6$, while the maximum value of $V_{u}$ is achieved when $P_{1}=2$ and $P_{2}=1.7$.

Similarly, the results of $V_{r}$ are as shown in Figures 14 and 15.

The relationship between $V_{r}$ and the design parameters of the palm is demonstrated in Figure 15. Without considering the palm design parameter $P_{2}, V_{r}$ gradually decreases as $P_{1}$ increases, when the decreasing speed tends to be constant. If the value of $P_{1}$ is $2, V_{r}$ can reach
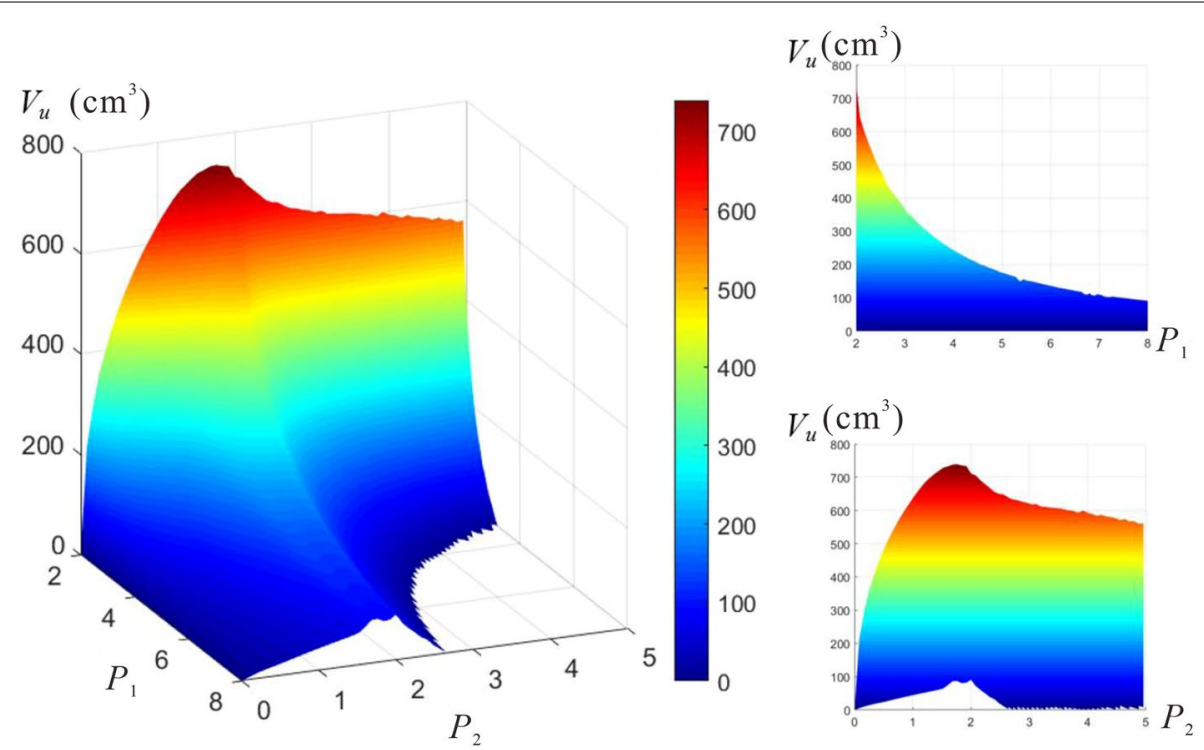

Figure 13 Volume of the workspace of the under-actuated finger 2 


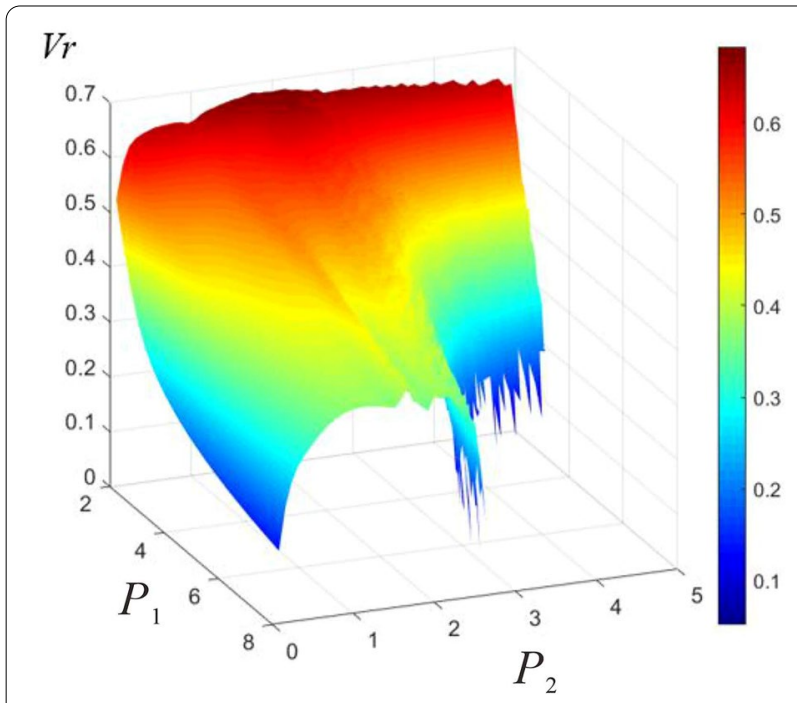

Figure 14 Stereogram of the ratio $V_{r}$ $\sim 0.68$, and the minimum value can be $\sim 0.53$. If the value of $P_{1}$ is 8 , the maximum value of $V_{r}$ can be $\sim 0.4$, and the minimum value can be only $\sim 0.15$. It can be observed that the palm design parameter, $P_{1}$ has a more significant influence on $V_{r}$. If the $P_{1}$ is small, the relationship between $V_{r}$ and the palm design parameter, $P_{2}$ is insignificant. As $P_{2}$ increases, the $V_{r}$ value tends to be stable. If the $P_{1}$ is large, the relationship between $V_{r}$ and $P_{2}$ becomes gradually apparent, showing a trend of increasing first, then stabilizing, and then decreasing as the $P_{2}$ increases.

If $P_{1}$ ranges from 2 to 4 , and $P_{2}$ ranges from 0.5 to 2.5 , the corresponding area color in the figure is dark red, which means that in this area, $V_{r}$ can exceed 0.5. For the dexterous hand developed by Borràs and Dollar [32], reasonable design parameters could reduce the negative impact of under-actuation, which leads to a reduction of
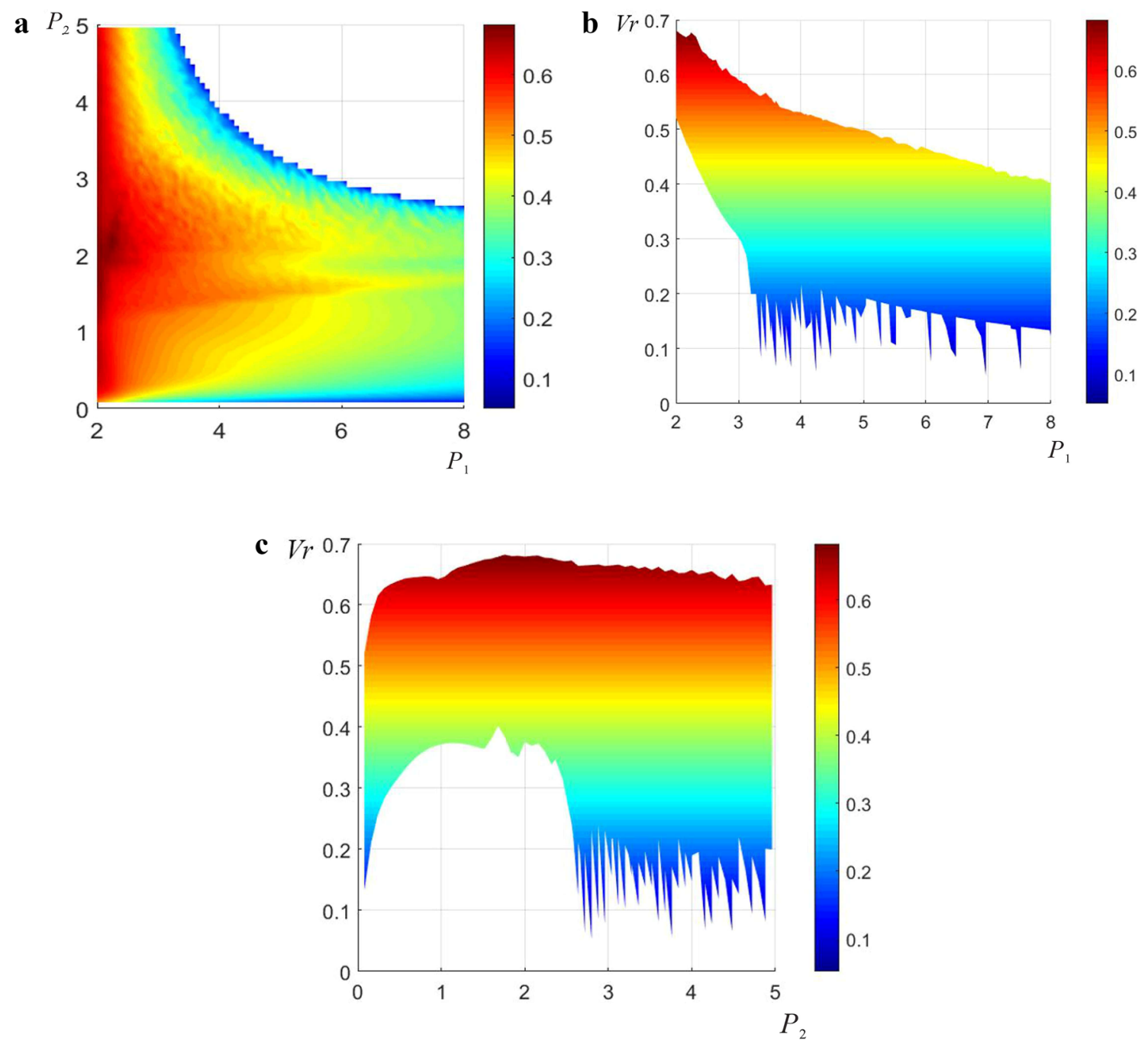

Figure $15 V_{r}$ projection: $\mathbf{a}$ on the $x O y$ plane, $\mathbf{b}$ on the $z O x$ plane, and (c) on the $y O z$ plane 
only $50 \%$ of the full-actuated hand workspace. Nevertheless, the optimal design parameters of the metamorphic hand in this study could achieve massive progress in the under-actuated finger workspace, and the maximum ratio between the workspace of under-actuated and that of full-actuated fingers could reach up to $68 \%$. This result undoubtedly proves once again that the introduction of metamorphic palms dramatically enhances the flexibility of dexterous hands. Introducing the corresponding mechanism in the palm is a novel idea for improving the overall flexibility of the dexterous hand.

\section{Conclusions}

We presented the dimensional synthesis of a novel metamorphic hand for maximal workspace, and obtained some valuable results:

(1) For the flat five-bar metamorphic palm, the symmetrical distribution of the links has a pronounced influence on the palm workspace. If the palm has a symmetrical structure, the optimal workspace of the palm can be obtained.

(2) By adjusting the proportional relationship between the links of the palm, the workspace of the palm can be maximized, and the average workspace can reach a maximum. The nondimensional design parameter, $P_{2}$ has a more significant impact on the metamorphic palm workspace; in the case of $P_{2}=1$, the metamorphic palm workspace can reach the maximum value, and the design parameter, $P_{1}$ has less influence on the metamorphic palm workspace.

(3) The metamorphic character of the flat five-bar palm allows the under-actuated finger to achieve a larger workspace, reaching $60 \%-70 \%$ of the full-actuated finger workspace. This confirms the application of the metamorphic mechanism on the palms of dexterous hands.

\section{Acknowledgements \\ The authors sincerely thank Ruiqin Wang of Tianjin University for his critical discussion and reading during manuscript preparation.}

\section{Authors' Information}

Wei An, born in 1996, is currently a Master candidate at Tianjin University, China. His research interests include metamorphic hands and robotics. E-mail: van2yun@tju.edu.cn.

Jun Wei, born in 1985, was a PhD candidate at Tianjin University, China. He received his doctoral degree from Tianjin University, China, in 2020. His research interests include Lie Group and reconfigurable mechanisms. E-mail: weijun@tju.edu.cn.

Xiaoyu Lu, born in 1995, received his Master's degree from Tianjin University, China, in 2020. His research interests include metamorphic hands and robotics. E-mail: Ixy_0826@tju.edu.cn.

Jian S. Dai, born in 1954, ASME Fellow and IMechE Fellow, is currently a professor in MOE Key Lab for Mechanism Theory and Equipment Design, International Centre for Advanced Mechanisms and Robotics, Tianjin University, PR China and the Centre for Robotics Research, School of Natural Sciences and Mathematics, King's College London, University of London, United Kingdom. His research interests include theoretical and computational kinematics, reconfigurable mechanisms, and multi-fingered robotic hands, robotics, advanced machinery, motion control, assembly, and industrial automation. E-mail: jian.dai@kcl.ac.uk.

Yanzeng Li, born in 1995, received his Master of Science degree from Rutgers University, US, in May, 2020. His research interests include big data analysis and applied machine learning methods. E-mail:yl1271@sph.rutgers.edu.

\section{Authors' Contributions}

JSD and XL were in charge of the whole trial; WA wrote the manuscript; $J W$ assisted with polishing this paper. YL helped in concluding the data. All authors read and approved the final manuscript.

\section{Funding}

Supported by National Natural Science Foundation of China (Grant No. 51535008).

\section{Competing Interests}

The authors declare no competing financial interests.

\section{Author Details}

${ }^{1}$ MOE Key Laboratory for Mechanism Theory and Equipment Design, International Centre for Advanced Mechanisms and Robotics, Tianjin University, Tianjin, China. ${ }^{2}$ Advanced Kinematics and Reconfigurable Robotics Lab, School of Natural and Mathematical Sciences, King's College London, London, UK.

${ }^{3}$ Rutgers, The State University of New Jersey, New Brunswick, New Jersey, USA.

Received: 30 January 2020 Revised: 31 January 2021 Accepted: 23 April 2021

Published online: 07 May 2021

\section{References}

[1] M Luo, T Mei, X Wang, et al. Grasp characteristics of an underactuated robot hand. IEEE International Conference on Robotics and Automation, 2004, Proceedings ICRA'04. 2004. IEEE, 2004, 3: 2236-2241. https://doi.org/ 10.1109/ROBOT.2004.1307394.

[2] MT Mason, J K Salisbury, J K Parker. Robot hands and the mechanics of manipulation. MIT Press, Cambridge, USA, 1985: 3-93.

[3] TOkada. Computer control of multijointed finger system for precise object-handling. IEEE Transactions on Systems, Man, and Cybernetics, 1982, 12(3): 289-299.

[4] MT Mason. The mechanics of manipulation. Proceedings of 1985 IEEE International Conference on Robotics and Automation, IEEE, 1985, 2: 544-548. https://doi.org/10.1109/ROBOT.1985.1087242.

[5] SC Jacobsen, E Iversen, D F Knutti, et al. Design of the Utah/MIT dextrous hand. Proceedings of 1986 IEEE International Conference on Robotics and Automation, IEEE, 1986, 3: 1520-1532. https://doi.org/10.1109/ROBOT. 1986.1087395.

[6] RTomovic, G Bekey, W J Karplus. A strategy for grasp synthesis with multifingered robot hands. Proceedings of 1987 IEEE International Conference on Robotics and Automation, IEEE, 1987, 4: 83-89. https://doi.org/10.1109/ ROBOT.1987.1087842.

[7] N Fukaya, SToyama, T Asfour, et al. Design of the TUAT/Karlsruhe humanoid hand. Proceedings of 2000 IEEE/RS International Conference on Intelligent Robots and Systems (IROS 2000)(Cat. No. 00CH37113), IEEE, 2000, 3: 1754-1759.

[8] J Dietrich, G Hirzinger, J Heindl, et al. Multisensory telerobotic techniques. In: Traditional and Non-Traditional Robotic Sensors. Springer, Berlin, Heidelberg, 1990: 255-283.

[9] J Ueda, Y Ishida, M Kondo, et al. Development of the NAIST-Hand with vision-based tactile fingertip sensor. Proceedings of the 2005 IEEE International Conference on Robotics and Automation, IEEE, 2005: 2332-2337.

[10] F Lotti, P Tiezzi, G Vassura. UBH3: Investigating alternative design concepts for robotic hands. Proceedings World Automation Congress, IEEE, 2004, 15: 135-140. 
[11] G Li, H Huang, H Guo, et al. Design, analysis and control of a novel deployable grasping manipulator. Mechanism and Machine Theory, 2019, 138: 182-204.

[12] C Zhao, H Guo, R Liu, et al. Actuation distribution and workspace analysis of a novel 3 (3RRIS) metamorphic serial-parallel manipulator for grasping space non-cooperative targets. Mechanism and Machine Theory, 2019, 139: 424-442.

[13] X Jin, Y Fang, D Zhang, et al. Design of dexterous hands based on parallel finger structures. Mechanism and Machine Theory, 2020, 152: 103952.

[14] X Jin, Y Fang, D Zhang, et al. Synthesis of 3-[P][S] parallel mechanisminspired multimode dexterous hands with parallel finger structure. Journal of Mechanical Design, 2020, 142(8).

[15] J Y Liu, R Zhao, X H Jia, et al. Underactuated dexterous hand with independent control of finger joint. Journal of Mechanical Engineering, 2020, 55(3): 47-55. (in Chinese)

[16] M Laffranchi, N Boccardo, S Traverso, et al. The Hannes hand prosthesis replicates the key biological properties of the human hand. Science Robotics, 2020, 5(46).

[17] D Gong, L Hao, J Yu, et al. Bionic design of a dexterous anthropomorphic hand actuated by antagonistic PAMs. 2020 IEEE International Conference on Real-time Computing and Robotics (RCAR), IEEE, 2020: 493-498. https:// doi.org/10.1109/RCAR49640.2020.9303299.

[18] R Wang, H Huang, R Xu, et al. Design of a novel simulated "soft" mechanical grasper. Mechanism and Machine Theory, 2021, 158: 104240.

[19] D Wang, Y Xiong, B Zi, et al. Design, analysis and experiment of a passively adaptive underactuated robotic hand with linkage-slider and rack-pinion mechanisms. Mechanism and Machine Theory, 2021, 155: 104092.

[20] H Yang, G Wei, L Ren, et al. A low-ccost linkage-spring-sendon-integrated compliant anthropomorphic robotic hand: MCR-Hand III. Mechanism and Machine Theory, 2021, 158: 104210.

[21] Y Yang, XXXiao, Z J Nan, et al., Design and kinematic characteristics of rigid-flexible coupling bionic finger. Journal of Mechanical Engineering, 2019, 55(11): 105-113. (in Chinese)

[22] C Luo, J W Su, W Z Zhang. Development of a co-circular slider linear-parallel and self-adaptive robot hand. Robot, 2019, 41: 519-525. (in Chinese)

[23] C Luo, S Yang, W Zhang, et al. MPJ hand: a self-adaptive underactuated hand with flexible fingers of multiple passive joints. 2016 International
Conference on Advanced Robotics and Mechatronics (ICARM), IEEE, 2016: 184-189. https://doi.org/10.1109/ICARM.2016.7606916.

[24] D Liang, J Song, W Zhang, et al. PASA hand: a novel parallel and selfadaptive underactuated hand with gear-link mechanisms. International Conference on Intelligent Robotics and Applications, Springer, Cham, 2016: 134-146.

[25] Q Jiang, S Song, W Zhang. A novel parallel and self-adaptive robot hand with triple-shaft pulley-belt mechanisms. International Conference on Intelligent Robotics and Applications, Springer, Cham, 2017: 752-763.

[26] L A T Al Abeach, S Nefti-Meziani, S Davis. Design of a variable stiffness soft dexterous gripper. Soft Robotics, 2017, 4(3): 274-284.

[27] P Glick, S A Suresh, D Ruffatto, et al. A soft robotic gripper with geckoinspired adhesive. IEEE Robotics and Automation Letters, 2018, 3(2): 903-910.

[28] J S Dai, D Wang. Geometric analysis and synthesis of the metamorphic robotic hand. Journal of Mechanical Design, 2007, 129(11): 1191-1197.

[29] J S Dai, D Wang, L Cui. Orientation and workspace analysis of the multifingered metamorphic hand-Metahand. IEEE Transactions on Robotics, 2009, 25(4): 942-947.

[30] M Ciocarlie, P Allen. A constrained optimization framework for compliant underactuated grasping. Mechanical Sciences, 2011, 2(1): 17-26.

[31] M Ciocarlie, F M Hicks, S Stanford. Kinetic and dimensional optimization for a tendon-driven gripper. 2013 IEEE International Conference on Robotics and Automation, IEEE, 2013: 2751-2758. https://doi.org/10.1109/ICRA. 2013.6630956

[32] J Borràs, A M Dollar. Dimensional synthesis of three-fingered robot hands for maximal precision manipulation workspace. The International Journal of Robotics Research, 2015, 34(14): 1731-1746.

[33] R K Hota, C S Kumar. Effect of hand design and object size on the workspace of three-fingered hands. Mechanism and Machine Theory, 2019, 133: 311-328.

[34] X Cui, J Sun, X S Zhang, et al. A metamorphic hand with coplanar reconfiguration. 2018 International Conference on Reconfigurable Mechanisms and Robots (ReMAR), IEEE, 2018: 1-7. https://doi.org/10.1109/REMAR.2018. 8449904.

\section{Submit your manuscript to a SpringerOpen ${ }^{\circ}$ journal and benefit from:}

- Convenient online submission

- Rigorous peer review

- Open access: articles freely available online

- High visibility within the field

Retaining the copyright to your article

Submit your next manuscript at $\boldsymbol{\nabla}$ springeropen.com 